\title{
Generation of Nanosized Structures on Ni and Fe Surfaces by Electron Irradiation
}

\author{
Keisuke Niwase ${ }^{1}$ and Hiroaki $\mathrm{Abe}^{2, *}$ \\ ${ }^{1}$ Department of Physics, Hyogo University of Teacher Education, Yashiro, Hyogo 673-1494, Japan \\ ${ }^{2}$ Japan Atomic Energy Research Institute-Takasaki, Takasaki 370-1292, Japan
}

We report a new type of self-organized nanostructure for pure $\mathrm{Ni}$ and Fe thin foils irradiated with $400 \mathrm{keV}$ electrons. Nanostructure develops at the electron exit surface of the foils. For Ni(001) irradiated along [001] direction at $105 \mathrm{~K}$ nanogrooves extend along [100] and [010] in the outer area of the beam, but the pattern differs from those near the beam center. For [011] irradiation, nanogrooves extend mainly along [100] direction and nanowires are finally formed. For Fe(111) irradiated along [111] at $300 \mathrm{~K}$ nanoholes are generated in the initial stage and then develop to a pattern of nanogrooves. The nanostructure is rather stable at temperatures lower than $823 \mathrm{~K}$, although the evaporation from the specimen surface occurs.

(Received October 22, 2001; Accepted December 13, 2001)

Keywords: nickel, iron, electron irradiation, transmission electron microscopy, self-organization, nanostructure

\section{Introduction}

Generation of nanosized structures is technologically important and self-organization process is one of the methods to produce regular structures. Several self-organization phenomena of defect clusters under high energy particle irradiations, such as voids, bubbles and stacking fault tetrahedra have been reported so far. ${ }^{1,2)}$ Recently, utilizing electron irradiation Niwase and coworkers have discovered a new type of self-organized nanostructure formation on the exit surface of thin gold foils irradiated with high doses of 360-1250 keV electrons at temperatures of about $100 \mathrm{~K} .{ }^{3)}$ The structure consists of aligned nanogrooves which develop parallel to the surface, and nanoholes and hillocks which grow parallel to the electron beam. The nanogrooves show strong irradiationdirection dependencies in their growth. They grow along the [100] and [010] directions for the [001] irradiation (Fig. 1), along [100] for the [011] irradiation, whereas no marked grooves are formed for the [111] irradiation. The sizes of nanogrooves and holes are between about 1 and $2 \mathrm{~nm}$, which are the smallest ones generated on metal surfaces so far. The formation of the pattern of grooves also has a surface orientation dependence. ${ }^{4)}$ The final structures of the thin foils under electron irradiation are nanoparticles or nanowires. This method has been utilized to produce long gold nanowires for investigations of the interesting physics such as the electron transport properties and the multi-shell structure. ${ }^{5,6)}$ Furthermore, the self-organized structures for silver and copper are investigated. ${ }^{7,8)}$

The formation of nanoholes and nanogrooves basically originates in the sputtering at the electron exit surface. But the anisotropic growth of the nanogrooves and nanoholes should be attributed to the irradiation-induced anisotropical flow of point defects. ${ }^{3,4,7,8)}$ To clarify the formation mechanism of the nanostructures, systematic investigations are needed. In this paper, we will show the formation of nanostructure for nickel and iron thin foils, that is rather different from those in

*Present address: Research Center for Nuclear Science and Technology, The University of Tokyo, Tokai-mura, Ibaraki 319-1188, Japan. the gold, silver and copper, especially in iron.

\section{Experimental}

In the present study, we used the wedge-shaped specimens produced from $99.998 \% \mathrm{Ni}$ and $99.997 \% \mathrm{Fe}$. After eliminating lattice defects by annealing, they were thinned by jet-polishing. The electron irradiations were performed using an electron beam of a diameter of several hundreds of nanometers in a JEOL 4000FX transmission electron microscope equipped with GATAN liquid nitrogen cooling stage, and observations were made in situ. The irradiation flux was about $2 \times 10^{24}$ electrons $\mathrm{m}^{-2} \mathrm{~s}^{-1}$. Grains with surface orientations nearly parallel to the $\{100\}$ or $\{111\}$ plane are selected and irradiated in several directions by tilting the specimen. Although the surface orientation and observation directions were not perfectly aligned with the crystallographic orientations in question, such crystallographic indices are used hereafter to characterize the surface orientation and observation directions.

\section{Results and Discussion}

\subsection{Nickel}

Figure 2 shows a nanostructure which appeared for a $\mathrm{Ni}(001)$ foil irradiated with $400 \mathrm{keV}$ electrons along [001] direction at $105 \mathrm{~K}$ to a dose of $7.0 \times 10^{27} \mathrm{e}^{-} \mathrm{m}^{-2}$. The diameter of an electron beam utilized for the irradiation was about $200 \mathrm{~nm}$, of which center is located near the center of Fig. 2(a). The micrograph was taken under a kinematic and slightly under-focus (the so-called void contrast) condition. In an area far from the beam center, we can find bright spots and bright images which connect the bright spots and extend along the [100] and [010] directions, as seen in a magnified view of Fig. 2(b). The pattern of nanogrooves in the area far from the beam center is similar to that of Au shown in Fig. 1. Stereomicroscopy revealed that the bright spots and the extended images correspond to nanoholes growing along the electron beam and nanogrooves developed parallel to the 

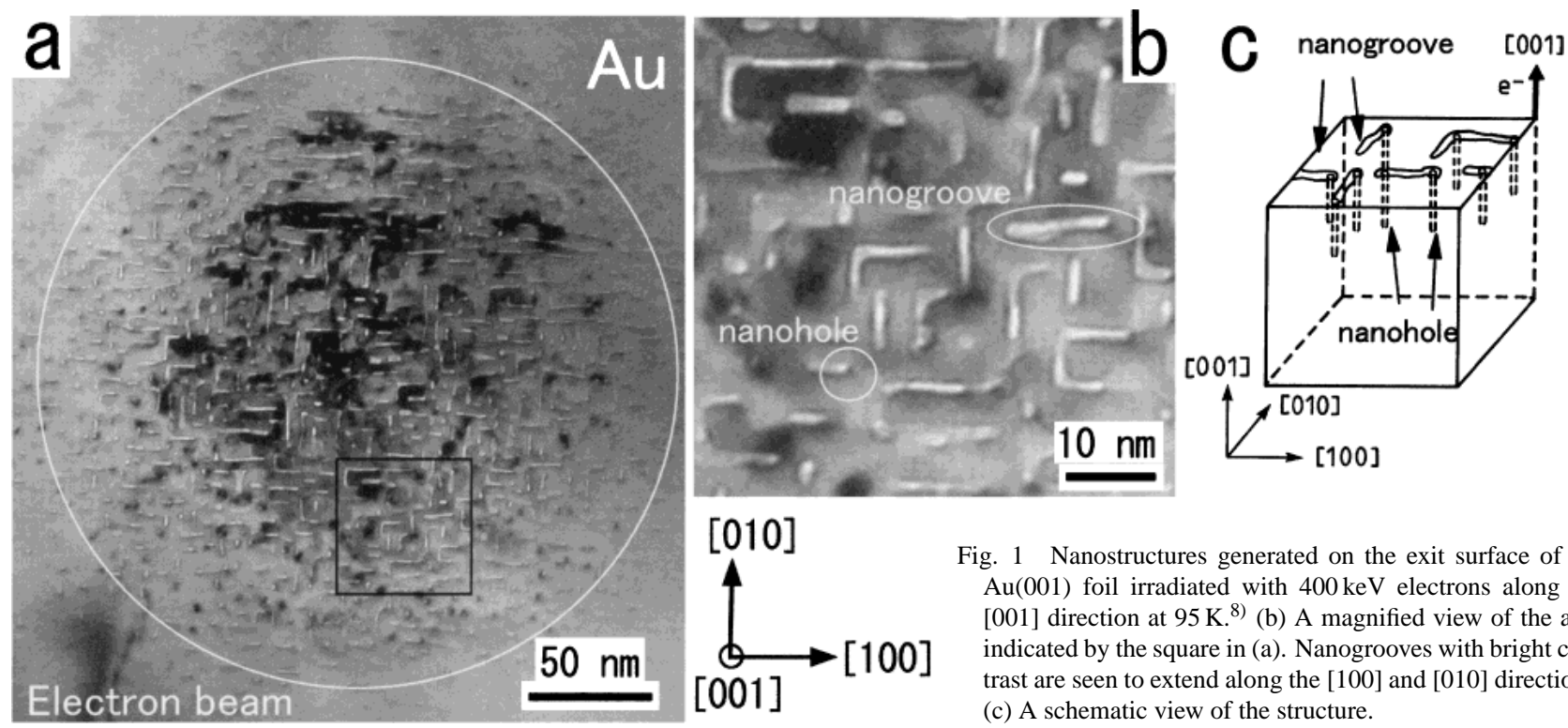

Fig. 1 Nanostructures generated on the exit surface of the $\mathrm{Au}(001)$ foil irradiated with $400 \mathrm{keV}$ electrons along the [001] direction at $95 \mathrm{~K}^{8}{ }^{8}$ (b) A magnified view of the area indicated by the square in (a). Nanogrooves with bright contrast are seen to extend along the [100] and [010] directions. (c) A schematic view of the structure.

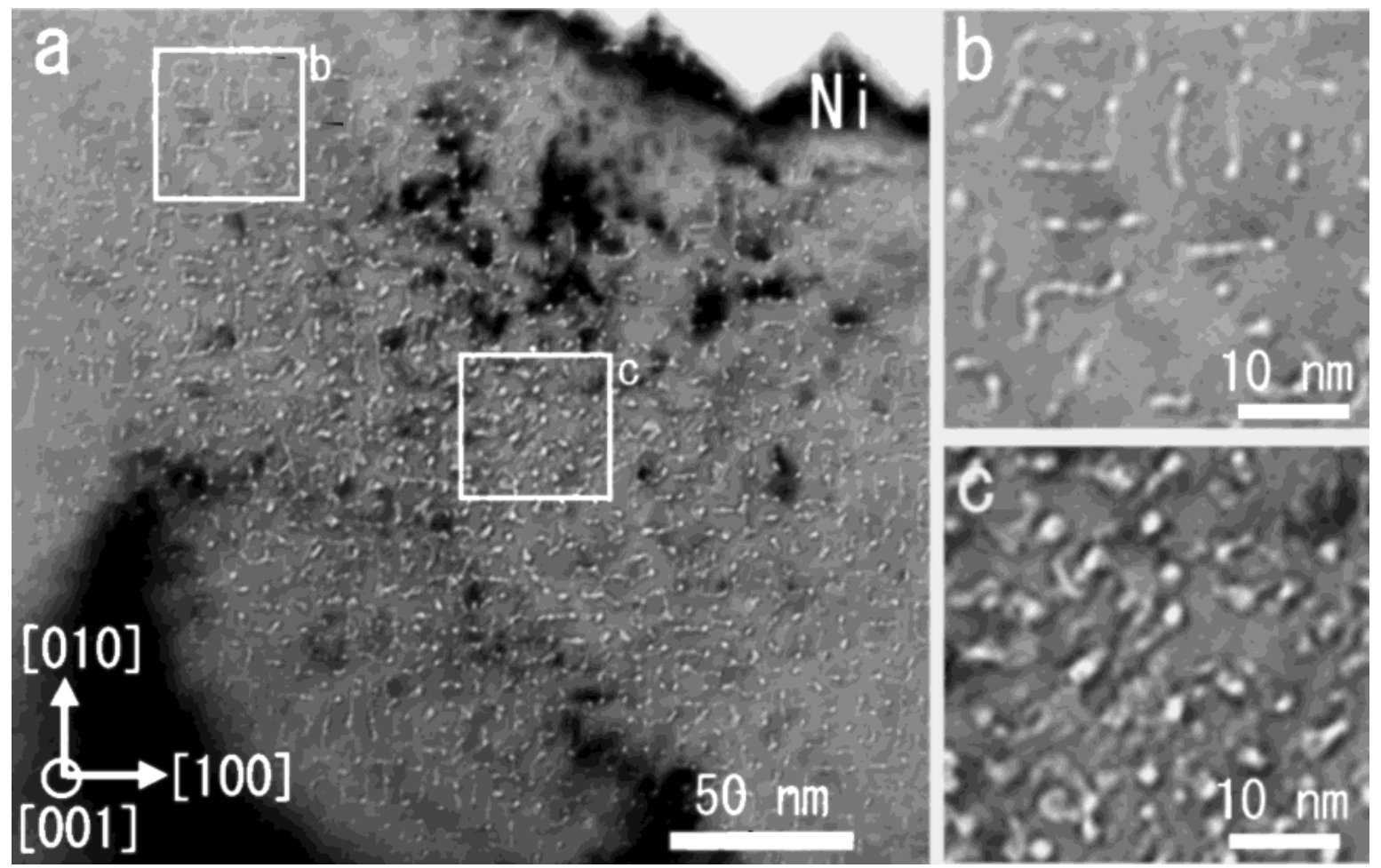

Fig. 2 Nanostructures generated on the exit surface of the Ni(001) foil irradiated with $400 \mathrm{keV}$ electrons along the [001] direction at $105 \mathrm{~K}$ to a dose of $7.0 \times 10^{27} \mathrm{e}^{-} \mathrm{m}^{-2}$. (b) A magnified view of the outer area of the electron beam. Bright spots are nanoholes. Nanogrooves are seen to extend along the [100] and [010] directions, connecting the nanoholes. (c) A magnified view of the area near the beam center. A slight extension of bright images along the [110] and [11 10$]$ directions can be seen.

exit surface, respectively. The width of grooves is about 1$2 \mathrm{~nm}$, similar to those for $\mathrm{Au}$ and $\mathrm{Ag} .{ }^{3,4,7,8)}$ The pattern near the center of the electron beam is rather different from that in the outer area as shown in Fig. 2(c). The extension of bright images along [100] and [010] directions is not observed but they extend slightly along [110] and [110] instead, of which pattern near the center of the electron beam is similar to those of $\mathrm{Ag}$ and $\mathrm{Cu}{ }^{7,8)}$ The results for Ni mentioned above are different from those of $\mathrm{Au}$, where the pattern is almost the same in the whole area although the extension of grooves becomes longer near the beam center as seen in Fig. 1(a).

The Ni(001) specimen was tilted to the direction near [011] and a flesh area was irradiated obliquely with $400 \mathrm{keV}$ electrons along [011] direction at $105 \mathrm{~K}$ to a dose of $8.4 \times$ $10^{27} \mathrm{e}^{-} \mathrm{m}^{-2}$. Bright images of nanogrooves tend to extend along [100] as shown in Fig. 3(a), similar to the cases of $\mathrm{Au}, \mathrm{Ag}$ and $\left.\mathrm{Cu}^{8}{ }^{8}\right)$ The irradiation was prolonged to a dose of $4.2 \times 10^{28} \mathrm{e}^{-} \mathrm{m}^{-2}$, then the penetration of nanogrooves from the electron exit surface to the incident surface occurred as shown in Fig. 3(b). Large holes appeared near the center 


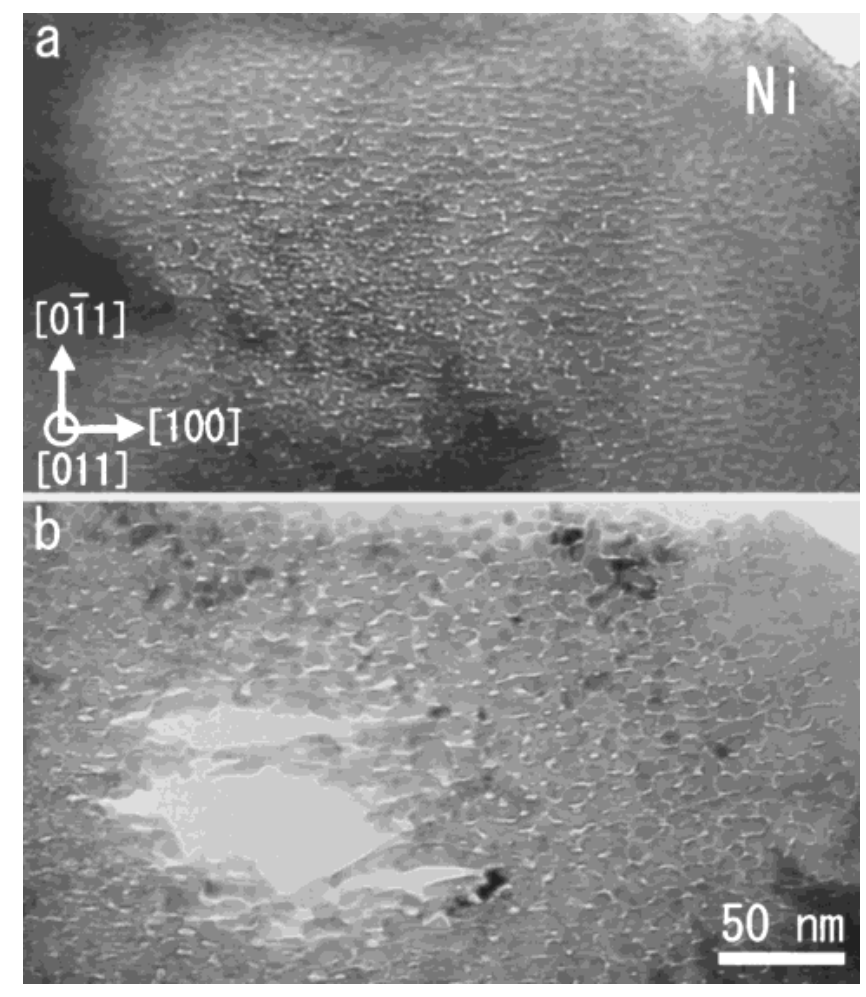

Fig. 3 Nanostructures generated on the exit surface of the Ni(001) foil irradiated with $400 \mathrm{keV}$ electrons along the [011] direction at $105 \mathrm{~K}$ to a dose of $8.4 \times 10^{27} \mathrm{e}^{-} \mathrm{m}^{-2}$. Nanogrooves tend to extend along the [100] direction. (b) is a micrograph taken after prolonged irradiation to a dose of $4.2 \times 10^{28} \mathrm{e}^{-} \mathrm{m}^{-2}$. Penetration occurred from the electron exit surface to the incident surface. Nanowires were formed reflecting the initial pattern.

of electron beam and several nanowires were generated. The nanowires tend to elongate along [100], reflecting the initial pattern of nanogrooves.

\subsection{Iron}

Figure 4 shows a nanostructure generated for a $\mathrm{Fe}(111)$ foil irradiated with $400 \mathrm{keV}$ electrons along [111] direction at $300 \mathrm{~K}$ to a dose of $4.4 \times 10^{28} \mathrm{e}^{-} \mathrm{m}^{-2}$. The micrograph was taken under a kinematic and slightly under-focus condition. The beam center is located near the edge of the specimen. The nanostructure produced by the electron irradiation can be roughly divided into two types; one is high density of nanoholes formed in the outer area of the electron beam as shown in a magnified view and the other is nanogrooves which appeared near the beam center. The diameter of nanoholes is about $2-4 \mathrm{~nm}$. The appearance of the two types of structure is due to the difference in the total dose as the beam intensity is stronger near the beam center. The irradiation dose described above is an average value. In situ observation has revealed that nanoholes are generated at first and then developed to nanogrooves with increasing the dose. Generation of high density of nanoholes under electron irradiation has not been observed for $\mathrm{Au}, \mathrm{Ag}$ and $\mathrm{Cu}^{3,4,7,8)}$ but has been reported for $\mathrm{Si}^{9 \text { ) }}$

One should note that nanostructure appeared at $300 \mathrm{~K}$ in the case of Fe. For Au, on the other hand, the nanostructure which appeared at $110 \mathrm{~K}$ became unstable on annealing at room temperature. Nanoholes are transformed into voids with larger diameters, while the hillocks decreased in height. ${ }^{3)}$ Then, to

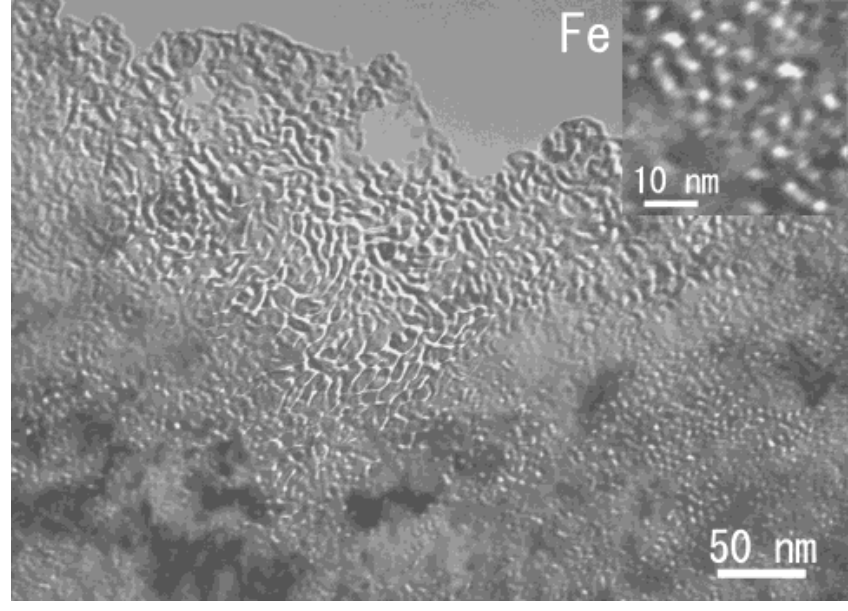

Fig. 4 Nanostructures generated on the exit surface of the Fe(111) foil irradiated with $400 \mathrm{keV}$ electrons along the [011] direction at $300 \mathrm{~K}$ to a dose of $4.4 \times 10^{28} \mathrm{e}^{-} \mathrm{m}^{-2}$. The pattern can be roughly divided into two types; one is nanogrooves which appear near the beam center and the other is high density of nanoholes formed in the outer area of the electron beam. A magnified view of the nanoholes is given in the inset.

know the stability of the nanostructure generated for Fe, the stepwise annealing was carried out with $50 \mathrm{~K} / 10 \mathrm{~min}$ steps from 373 to $823 \mathrm{~K}$ in the transmission electron microscope.

Figures 5(a) and (b) show the change of the images observed at 623 and $823 \mathrm{~K}$, respectively. The pattern scarcely changed up to $573 \mathrm{~K}$, but a clear change became observed above $623 \mathrm{~K}$; the steps denoted by arrows were observed (Figs. 5(a) and (b)). In spite of such an apparent change in the specimen surface, at $623 \mathrm{~K}$ we can still observe some nanogrooves and nanoholes whose size and pattern are similar to those before annealing as seen Fig. 4. At $823 \mathrm{~K}$, on the other hand, a remarkable change in the nanostructures becomes observed especially for the nanogrooves (Figs. 5(b)). However, there still remains some nanoholes whose diameters are almost the same as those before annealing. The result is different from that of Au where nanoholes were transformed into voids with larger diameters and the surface became smoother. The changes in Au suggest the effect of surface diffusion. ${ }^{3)}$ Therefore, we guess that thermal evaporation on Fe surface becomes remarkable to form the steps and to change the structure at temperature above $623 \mathrm{~K}$ and that material transport on Fe surface by surface diffusion is not so remarkable as to change the nanostructure below $823 \mathrm{~K}$. However, one should note that residual $\mathrm{O}_{2}$ gas in the conventional TEM may affect the generation or the behavior of nanostructure similarly to the case of silicon, ${ }^{9}{ }^{9}$ although the electron exit surface of Fe where the pattern developed should be rather clean due to sputtering. Then, further investigations on this point are desired.

\section{Conclusions}

The self-organized nanostructure formation under $400 \mathrm{keV}$ electron irradiation was investigated for $\mathrm{Ni}(001)$ and $\mathrm{Fe}(111)$ thin foils. It was found that nanostructure appears on the exit surface of the foil. For Ni irradiated along the [001] direction at $105 \mathrm{~K}$, nanogrooves of about $1-2 \mathrm{~nm}$ in width extend along the [100] and [010] directions in the outer area of 

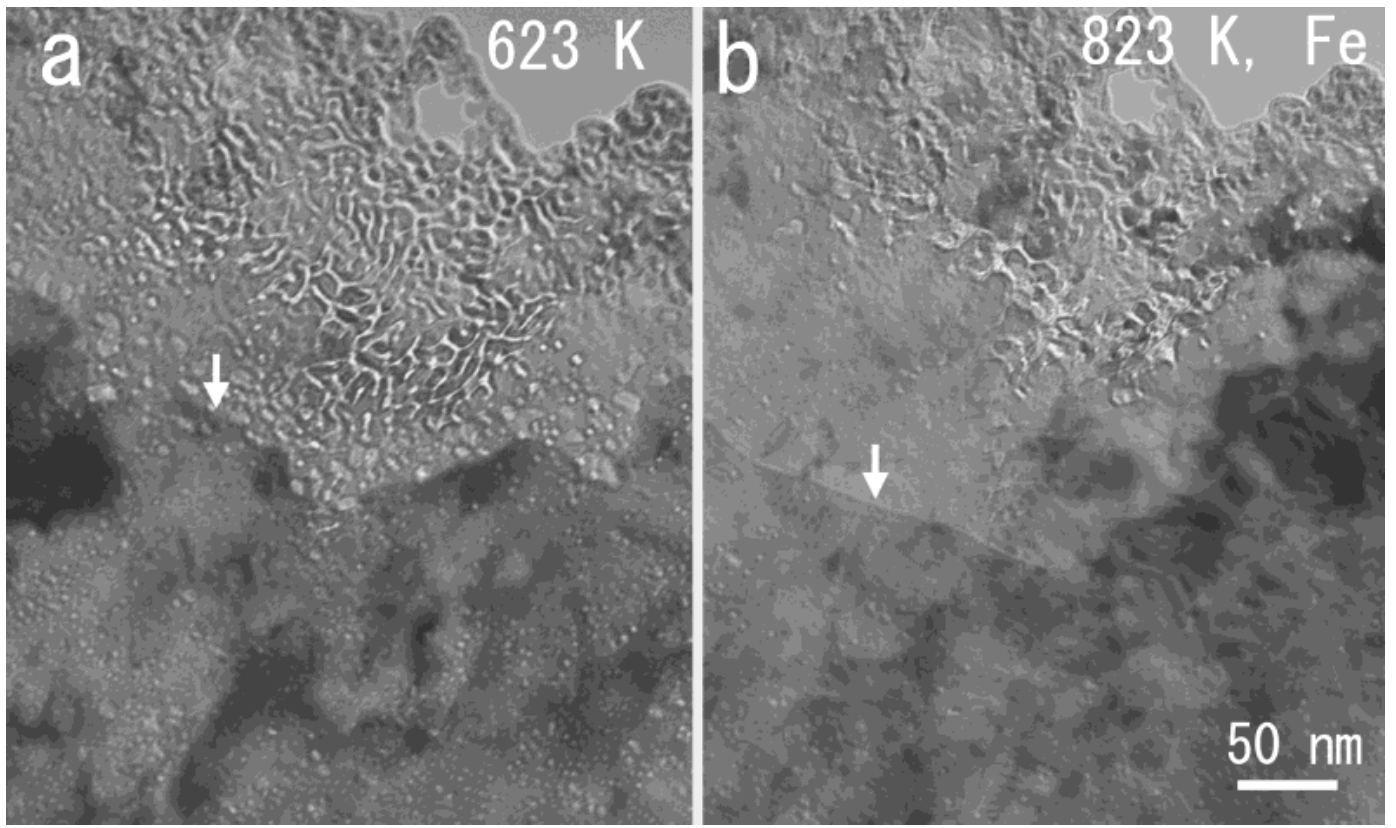

Fig. 5 The change of nanostructure of the $\mathrm{Fe}(111)$ foil on annealing at (a) $623 \mathrm{~K}$ and (b) $823 \mathrm{~K}$. The nanostructure was generated by the irradiation with $400 \mathrm{keV}$ electrons along the [011] direction at $300 \mathrm{~K}$ to a dose of $4.4 \times 10^{28} \mathrm{e}^{-} \mathrm{m}^{-2}$ as shown in Fig. 4. Steps which appeared on annealing are denoted by arrows in (a) and (b).

the beam, while near the beam center the pattern is different. Nanogrooves tend to extend along the [100] direction for the [011] irradiation and nanowires were finally formed. For Fe irradiated at room temperature, nanoholes of about $2-4 \mathrm{~nm}$ in diameter appeared at first and then developed to nanogrooves. On annealing up to $823 \mathrm{~K}$, changes of the nanostructure due to surface diffusion were scarcely observed, although thinning of the specimen by evaporation occurred. This type of self-organization phenomena may be interesting in the view point of the nanostructural fabrication.

\section{REFERENCES}

1) A. Seeger, N. Y. Jin, F. Phillipp and M. Zaiser: Ultramicroscopy 39 (1991) 342-354.

2) W. Joeger and H. Trinkaus: J. Nucl. Mater. 205 (1993) 394-410.

3) K. Niwase, W. Sigle, F. Phillipp and A. Seeger: Phil. Mag. Lett. 74 (1996) $167-174$.

4) K. Niwase, W. Sigle, F. Phillipp and A. Seeger: J. Surface Analysis 3 (1997) 440-445.

5) Y. Kondo and K. Takayanagi: Phys. Rev. Lett. 79 (1997) 3455-3458.

6) Y. Kondo and K. Takayanagi: Science 289 (2000) 606-608.

7) K. Niwase, F. Phillipp, W. Sigle and A. Seeger: J. Electron Microsc. 48 (1999) 495-502.

8) K. Niwase, F. Phillipp and A. Seeger: Jpn. J. Appl. Phys. 39 (2000) 4624 4628.

9) S. Takeda, K. Koto, S. Iijima and T. Ichihasi: Phys. Rev. Lett. 79 (1997) 2994-2997. 